\title{
Uniaxial stress method for delicate crystals: Application to Shubnikov-de Haas and superconductivity studies in organic conductors
}

\author{
C. E. Campos and J. S. Brooks \\ Physics Department, Boston University, Boston, Massachusetts 02215 \\ P. J. M. van Bentum, J. A. A. J. Perenboom, and J. Rook \\ High Field Magnet Laboratory and Research Institute for Materials, University of Nijmegen, NL-6525 ED \\ Nijmegen, The Netherlands \\ S. J. Klepper \\ F. B. National Magnet Laboratory, Massachusetts Institute of Technology, Cambridge, Massachusetts 02139 \\ M. Tokumoto \\ Electrotechnical Laboratories, Tsukuba, Ibaraki 305, Japan
}

(Received 22 July 1994; accepted for publication 4 November 1994)

\begin{abstract}
A method to measure the resistance of fragile single crystals under high uniaxial stress is reported. To compensate for its natural brittleness, the crystal is embedded in epoxy, whose shape is optimized to sustain the stress. This method was used to study the magnetoresistance of $(\mathrm{BEDT}-\mathrm{TTF})_{2} \mathrm{KHg}(\mathrm{SCN})_{4}$ at low temperatures and high magnetic fields and the superconducting transition temperature of (BEDT-TTF $)_{2} \mathrm{Cu}(\mathrm{NCS})_{2}$ under uniaxial stress perpendicular to the planes of BEDT-TTF molecules. The results obtained indicate a high degree of homogeneity of the stress in the crystal and comparison with previous hydrostatic pressure measurements reveal the true uniaxial nature of the stress applied. (C) 1995 American Institute of Physics.
\end{abstract}

\section{INTRODUCTION}

The application of pressure to a single crystal of a conductive material tunes its crystal structure, which in turn modifies its band structure and Fermi surface. These changes provide an important check on the validity of the band structure calculations and, more importantly, often lead to new low-temperature phase transitions. In particular, members of the (BEDT-TTF) ${ }_{2} \mathrm{X}$ (where $\mathrm{X}$ is one of a number of anion structures) family of quasi-two-dimensional organic conductors ${ }^{1}$ have proven to be very sensitive to pressure. Mydrostatic pressure studies on the (BEDT-TTF) ${ }_{2} \mathrm{MHg}(\mathrm{SCN})_{4}$ $\left(\mathrm{M}=\mathrm{NH}_{4}, \mathrm{~K}, \mathrm{Tl}\right.$, or $\mathrm{Rb}$ ) have shown that the Fermi surface of these materials changes by as much as $1 \% / \mathrm{kbar}$ (for regular metals this change is of the order of $0.1 \% / \mathrm{kbar}$ ), and their density wave or superconducting ground state is suppressed with a few kbar of applied pressure. ${ }^{2}$ These enhanced pressure effects are due to the high compressibility of molecular crystals and the anisotropy of their structure. The crystal structure of the (BEDT-TTF $)_{2} \mathrm{MHg}(\mathrm{SCN})_{4}$ group consists of two-dimensional layers of conductive BEDT-TTF molecules alternating with $\mathrm{MHg}(\mathrm{SCN})_{4}$ insulating sheets. ${ }^{3}$ Electronic transport takes place on the planes of molecules and changes in either the spacing between planes or in the relative positions of the molecules within a plane result in the observed pressure dependent phenomena. The effect of hydrostatic pressure is a compression of both interplane and intraplane unit cell parameters, whereas uniaxial stress decreases the distance between planes while increasing the in-plane lattice parameters. Combining the studies of the effects of both types of pressure allows the separation of the contributions of inter- and intraplane interactions to the transport properties.
Whenever sturdy and flat faced single crystals are available, uniaxial stress studies can be easily performed by applying stress directly on the bare crystal, as was recently done on high- $T_{c}$ superconductors. ${ }^{4}$ In the case of organic conductors, the delicate nature of the single crystals available hindered all previous attempts to study their uniaxial stress properties. Tokumoto et al. ${ }^{5}$ applied uniaxial stress directly on a bare crystal of (BEDT-TTF $)_{2} \mathrm{Cu}(\mathrm{NCS})_{2}$, a superconducting material with $T_{c}=11 \mathrm{~K}$, with an upper limit of $\approx 0.01 \mathrm{kbar}$. Kusuhara et al. ${ }^{6}$ applied tensile stress to a crystal of the same material by fixing two ends of the crystal to a substrate with smaller thermal contraction than (BEDT-TTF $)_{2} \mathrm{Cu}(\mathrm{NCS})_{2}$ and cooling the assembly. This method gave consistent results for several samples $\left(T_{c}\right.$ increased with tensile stress along the $b$ axis for both copper and quartz glass substrates) but is restricted by several factors: the amount of strain applied to the crystal is temperature dependent, is not easily estimated, and cannot be changed in situ. In this report we will describe a sample preparation method that allows the application of uniaxial stress (up to $\approx 5 \mathrm{kbar}$ ) to single crystals of organic conductors and the uniaxial stress apparatus used to apply continuous known values of stress at the low temperatures (down to $0.5 \mathrm{~K}$ ) and high magnetic fields (up to $30 \mathrm{~T}$ ) required to observe changes in the transport properties of these materials. The basic idea is to compensate for the brittle and irregular nature of the crystals by incasement in a medium whose shape can be optimized and whose physical properties closely match those of organic salts.

\section{SAMPLE PREPARATION}

Single crystals of organic conductors are obtained by slow deposition of the chemical composites on a platinum 


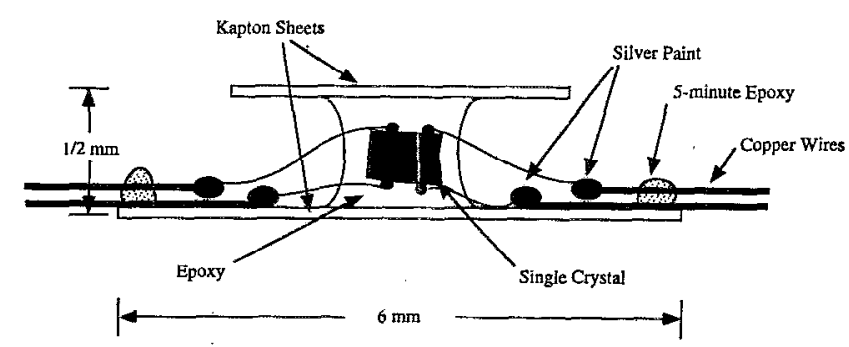

FIG. 1. Schematic diagram of the epoxy-crystal assembly (overall dimensions are noted in the figure but constituent parts are not drawn to scale). Details of the assembly procedure are described in the text.

electrode of an electrochemical cell. ${ }^{7}$ The resultant crystals have cubic or thin platelet shapes with dimensions on the order of tenths of a millimeter. The largest and flattest faces usually correspond to the orientation of the conducting planes of BEDT-TTF molecules. Since these crystals lack the necessary strength to support any but the slightest stress or shear, the solution to the problem of subjecting them to uniaxial stress resides in finding a suitable material that can support the crystal and transmit the stress to it along one single direction. The whole crystal is embedded in a cylinder of epoxy which is subsequently cured to solid hardness and then cooled and subjected to uniaxial stress. A similar method has been used to study the effect of uniaxial stress on the ac susceptibility of grain aligned high- $T_{c}$ superconductors ${ }^{8}$ but, to our knowledge, this is the first application of such method to the measurement of resistance in single crystals of organic conductors.

In order to improve the uniformity of the stress applied, we select small $\left(\approx 0.5 \times 0.5 \mathrm{~mm}^{2}\right.$ faces) and thin $(\leqslant 0.3 \mathrm{~mm})$ single crystals from the available batches. Two pairs of thin gold wires are attached to the opposite flat faces of the crystal with gold paint for the standard four terminal resistance measurements across the conducting BEDT-TTF layers. Separately, one $4 \times 4 \mathrm{~mm}^{2}$ and one $6 \times 6 \mathrm{~mm}^{2}$ square sheets of kapton are glued with $\mathrm{N}$ grease to the center of two glass microscope slides. A drop of epoxy ${ }^{9}$ slightly larger than the crystal is placed on the biggest kapton square and the crystal positioned on it with its flat faces parallel to the glass slide. A few more drops of epoxy are added until the crystal is fully covered. Two separators ( $0.5 \mathrm{~mm}$ thick flat copper plates) are placed on both sides of the assembly and the other glass slide is brought down until the epoxy forms a catenoidlike shape between the two kapton squares. A small weight is added on the top slide to lock the assembly together.

After the epoxy cures, ${ }^{10}$ the weight and the top glass slide are removed and 40 gauge copper wires ${ }^{11}$ are connected to each gold lead with silver paint and anchored to the bottom kapton square with 5-min epoxy. The final assembly is a solid cylindrical epoxy-sample tube that can sustain high uniaxial stress in the direction perpendicular to the crystal's conducting planes and has the four leads required for resistance measurements (see Fig. 1).

\section{APPARATUS DESIGN AND OPERATION}

The most important factor that constrains the design of a low-temperature uniaxial stress probe is the ability to trans-

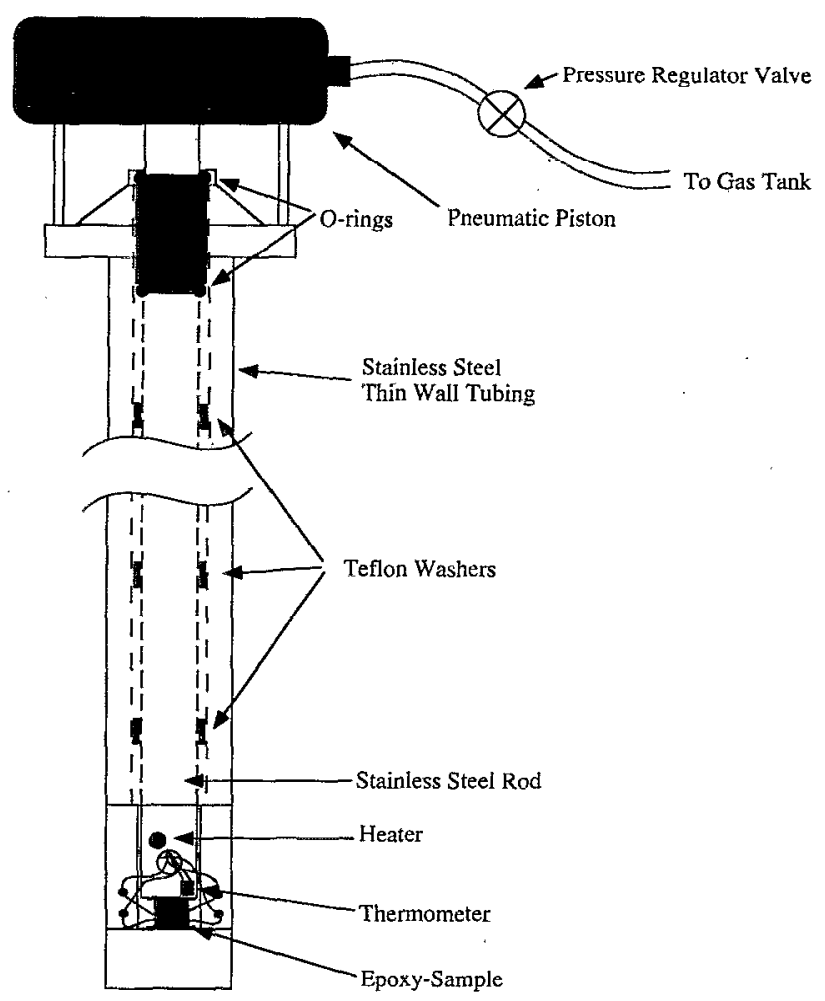

FIG. 2. Schematic diagram of the Uniaxial Stress Apparatus (constituent parts are not drawn to scale). The inset's diameter is small enough to fit through a vacuum seal into a standard $5 / 8 \mathrm{in}$. ${ }^{3} \mathrm{HL}$ fridge and in a $1 \mathrm{in}$. bore magnet.

mit a known stress down to the sample that can be changed continuously while minimizing losses to friction in the vacuum seals. We have chosen to implement a simple gas piston design where room-temperature gas pressure on the top of the piston is transmitted to the sample by a stainlesssteel rod (Fig. 2). The rod is hollow to minimize heat losses from the sample chamber and, to prevent bending or buckling, is guided down the cylinder by evenly spaced lubricated Teflon washers. Friction at the vacuum seal is reduced by placing two rubber o-rings at each outer end of a hollow lubricated cylinder that fits snugly around the rod. The rod is screwed to a commercial pneumatic piston, and the pressure in the piston controlled by a pressure regulator valve connected to a high pressure helium gas tank. The diameter of the piston is $63 \mathrm{~mm}$, and the epoxy-crystal cylinder diameter is usually around $2 \mathrm{~mm}$, which gives a pressure amplification factor of $\approx 1000$. Standard pneumatic pressure of 6 bar results in $6 \mathrm{kbar}$ applied to the sample. Higher pressure can be reached either by increasing the piston gas pressure (the pneumatic piston is rated for pressures up to 10 bar) or by reducing the size of the epoxy-crystal cylinder. The temperature is measured by a calibrated $\mathrm{RuO}_{2} 3 \mathrm{k} \Omega$ resistor glued to the end of the stainless-steel rod for good thermal contact with the sample and a $100 \Omega$ heater resistor is also attached to the piston for temperature control capability.

\section{EXPERIMENTAL RESULTS}

Our main interest is to study the Fermi surfaces of organic conductors and how they change with uniaxial stress 


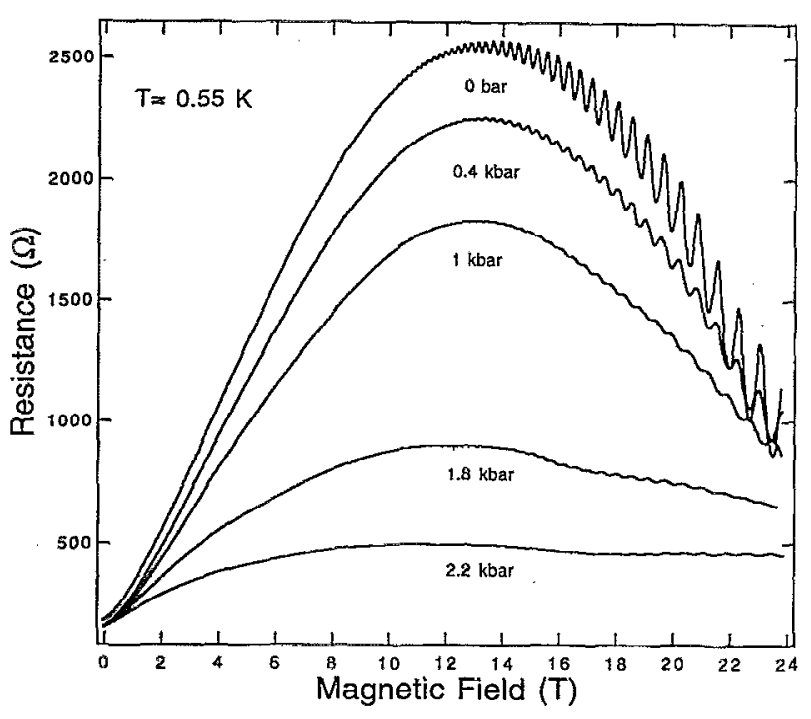

FIG. 3. Magnetoresistance of (BEDT-TTF) ${ }_{2} \mathrm{KHg}(\mathrm{SCN})_{4}$ at $T=0.55 \mathrm{~K}$ measured with ac lock-in detection. Uniaxial stress is perpendicular to the BEDT-TTF conduction planes (along the crystal $b$ axis).

by measuring magnetoresistance (MR) at low temperatures and high magnetic fields. We have selected two examples from our research program which demonstrate both the true uniaxial nature of the applied stress and the high degree of stress uniformity within the sample.

We chose to concentrate first on the uniaxial stress dependence of the magnetotransport properties of (BEDT-TTF $)_{2} \mathrm{KHg}(\mathrm{SCN})_{4}$ since a similar hydrostatic pressure study had been conducted previously by our group. ${ }^{2}$ Figure 3 shows typical MR data obtained at a temperature of $0.55 \mathrm{~K}$ in a resistive magnet for five different values of uniaxial stress along the crystal $b$ axis. Shubnikov-de Haas (SdH) oscillations superposed on a smooth MR background are clearly visible for all stresses (Figs. 3 and 4). Fourier analysis of the zero stress $\mathrm{SdH}$ signal reveals a single fundamental frequency peak at $F=(\hbar c / 2 \pi e) A=672 \pm 6 \mathrm{~T}$ (where $A$ is the extermal area of the cross section of the Fermi surface), in agreement with the atmospheric pressure value of $670 \mathrm{~T}$. This frequency is attributed to the hole closed orbit part of the Fermi surface and corresponds to approximately $16 \%$ of the area of the Brillouin zone. ${ }^{3}$ Upon application of uniaxial stress the frequency decreases linearly by 17.2 $\mathrm{T} / \mathrm{kbar}$, whereas hydrostatic pressure measurements found the frequency to increase with pressure [Fig. 4(a)]. The difference in the pressure dependence of the fundamental frequency is consistent with the type of pressure applied, since the frequency is proportional to the extermal area of the Fermi surface and therefore, to a first order approximation, proportional to the size of the Brillouin zone. Hydrostatic pressure compresses the crystal unit cell, thereby increasing the size of the Brillouin zone, whereas uniaxial stress applied along a direction perpendicular to the conducting planies expands the unit cell parameters along the plane (Poisson's effect), thereby decreasing the size of the Brillouin zone.

The homogeneity of the uniaxial stress applied to the crystal can be estimated from the relative amplitudes $\Delta R / R$
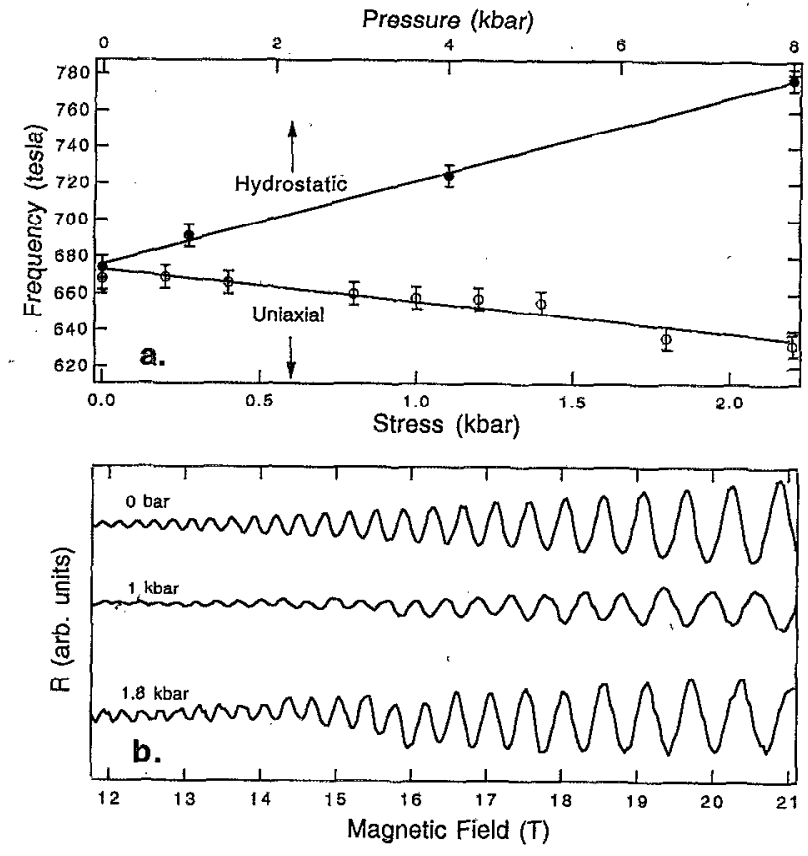

FIG. 4. (a) Variation of SdH frequency with hydrostatic pressure (top scale, from Ref. 2) and uniaxial stress (bottom scale) for $(\text { BEDT-TTF })_{2} \mathrm{KHg}(\mathrm{SCN})_{4}$. The slopes of the fitted lines are +12.1 and $-17.2 \mathrm{~T} / \mathrm{kbar}$, respectively. (b) Oscillatory part of the magnetoresistance (SdH signal). at $0.55 \mathrm{~K}$ for different uniaxial stresses (each curve is plotted on an independent resistance scale).

of the SdH oscillations for different stresses. Inhomogeneous strcss applicd to a crystal results in a spread of the extremal areas of the Fermi surface and, consequently, a spread in the $\mathrm{SdH}$ frequency. If the frequency spread follows a Lorentzian distribution there will be an amplitude reduction factor for the fundamental harmonic $R=\exp \left[-2 \pi(\Delta F)_{0} / H\right]$, where $2(\Delta F)_{0}$ is the full width at half-maximum of the Lorentzian curve. ${ }^{12}$ Assuming that at zero stress $(\Delta F)_{0}=0$ and that reductions in the amplitudes of the oscillations are due solely to inhomogeneity in the stress, we estimate that $2(\Delta F)_{0} / F$ is never worse than $2.5 \%$.

Last, we note that both the zero-field resistance and the MR maximum, which for (BE.DT-TTF $)_{2} \mathrm{KHg}(\mathrm{SCN})_{4}$ occurs around $14 \mathrm{~T}$, decrease with uniaxial stress, albeit at different rates. The decrease in zero-field resistance, which is due to an increase in the BEDT-TTF interplane wave-function overlap, is slower than the peak MR change, which is due to the suppression of the density wave ground state present in this material below $8 \mathrm{~K}$. Under hydrostatic pressures comparable effects are observed. ${ }^{2}$

The same technique was used to study the superconducting transition of (BEDT-TTF) ${ }_{2} \mathrm{Cu}(\mathrm{NCS})_{2}$, the organic conductor studied in previous uniaxial stress reports. Stress was applied along the crystal $a$ axis $\left(p_{a}\right)$, i.e., perpendicular to the BEDT-TTF conducting layers. Our results are shown in Fig. 5. The normal state resistivity again drops with uniaxial stress and $T_{c}$ decreases monotonically by about $d T_{c} / d p_{a}=-2 \mathrm{~K} / \mathrm{kbar}$ from a zero stress value of $11 \mathrm{~K}$. This value of $T_{c}$ at zero stress is identical to that measured on bare crystals from the same batch, but the width of the superconducting transition increased from $2 \mathrm{~K}$ on the bare 


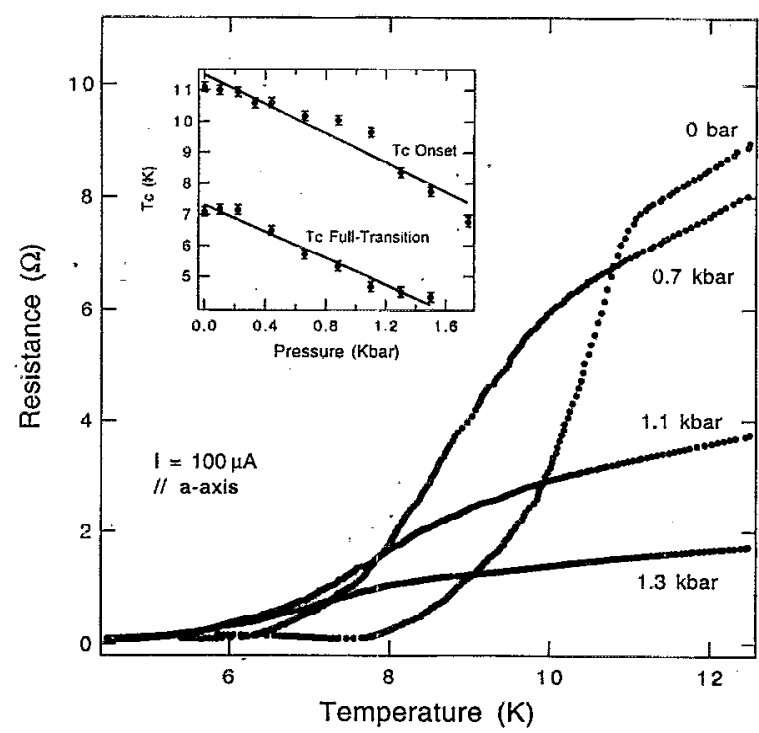

FIG. 5. Resistance of (BEDT-TTF $)_{2} \mathrm{Cu}(\mathrm{NCS})_{2}$ versus temperature as a function of uniaxial stress along the $a$ axis. Inset: superconducting transition temperature variation with stress. Slopes of the lines fitted to the onset and full-transition temperatures are -2.1 and $-2.0 \mathrm{~K} / \mathrm{kbar}$, respectively.

crystals to $4 \mathrm{~K}$ for the one cooled in epoxy (see Fig. 5 inset). Although this indicates that stress inhomogeneity can be induced in these very thin samples during the initial epoxy cure and/or during cooldown, we note that the superconducting transition width, $T_{c}$ onset- $T_{c}$ full transition, remains constant for all stresses, which shows that the stress subsequently applied to the crystal is homogeneous. Tokumoto et al. ${ }^{5}$ also observed a decrease in $T_{c}$ with stress applied to a bare crystal along the $a$ axis and the $a$ axis stress derivative of $T_{c}$ derived from the thermal expansion coefficient was also found to be negative, although at a higher rate of $d T_{c} / d p_{a}=-4.8 \mathrm{~K} / \mathrm{bar} .{ }^{13}$ Kusuhara et al. found $T_{c}$ to increase with stress, but their data cannot be compared directly to ours, since their method applies a tensile stress to the crystals along the $b$ axis. ${ }^{6}$

\section{DISCUSSION}

We have described a method to measure the resistance of single crystals of organic conductors under direct uniaxial stress. Its application to the study of transport in organic conductors yielded results consistent with previous hydrostatic pressure studies, with the advantage that the changes induced by hydrostatic pressure can be obtained with much lower uniaxial stress. Although the stress was always applied to the crystal's flat faces, small modifications in the sample preparation and in the design of the apparatus should allow the application of stress along the two other crystal directions while keeping the magnetic field perpendicular to the conducting planes. The technique would be extendable to other measurements, such as magnetization or specific heat, and other types of fragile single crystals, such as the Bechgaard salts.

\section{ACKNOWLEDGMENTS}

Work at Boston University is supported by NSF-DMR92-14889. The high-field work was carried out at the High Field Magnet Laboratory in the University of Nijmegen and at the Francis Bitter National Magnet Laboratory (supported by the NSF). C.E.C. is supported by a fellowship from Junta Nacional de Investigação Cientifica e Tecnológica and acknowledges the hospitality of the staff of the High Field Magnet Laboratory during his stay at the University of Nijmegen. The authors are grateful to Dr. Kinoshita, Dr. H. Anzai, and Dr. Y. Tanaka for providing the samples used in this study.

${ }^{1}$ For a recent review of this subject see, T. Ishiguro and $\mathrm{K}$. Yamaji, Organic Superconductors (Springer, Berlin, 1990).

${ }^{2}$ J. S. Brooks, C. C. Agosta, S. J. Klepper, M. Tokumoto, N. Kinoshita, H. Anzai, S. Uji, H. Aoki, A. S. Perel, G. J. Athas, and D. A. Howe, Phys. Rev. Lett. 69, 156 (1992); X. Chen, Ph.D. thesis, Boston University, Boston, MA, 1994 (unpublished).

${ }^{3}$ H. Mori, S. Tanaka, M. Oshima, G. Saito, T. Mori, Y. Maruyama, and $H$. Inokuchi, Bull. Chem. Soc. Jpn. 63, 2183 (1990).

${ }^{4}$ U. Welp, M. Grimsditch, S. Fleshler, W. Nessler, J. Downey, G. W. Crabtree, and J. Guimpel, Phys. Rev. Lett. 69, 2130 (1992).

${ }^{5}$ M. Tokumoto, K. Murata, N. Kinoshita, K. Yamaji, H. Anzai, Y. Tanaka, Y. Hayakawa, K. Nagasaka, and Y. Sugawara, Mol. Cryst. Liq. Cryst. 181, 295 (1990).

${ }^{6}$ H. Kusuhara, Y. Sakata, Y. Ueba, K. 'lada, M. Kaji, and T. Ishiguro, Solid State Commun. 74, 251 (1990).

${ }^{7}$ M. Oshima, H. Mori, G. Saito, and K. Oshima, Chem. Lett. 1989, 1159 (1989).

${ }^{8}$ Y. Motoi, K. Fujimoto, U. Uwe, and T. Sakudo, J. Phys. Soc. Jpn. 60, 384 (1991).

${ }^{9}$ We have used both Emerson and Cuming Stycast 2651 with catalyst $23 \mathrm{LV}$ and standard 5-min epoxy with similar experimental results. In general, samples mounted with 5-min epoxy have lower contact resistances but cannot withstand stresses as high as those mounted with Stycast 2651. Typical stress limits are 2.5-3.5 kbar for 5-min epoxy and 3-5 kbar for Stycast 2651.

${ }^{10}$ Epoxy was always cured at room temperature following the manufacturer's recommended curing schedule, since the samples can be damaged by heating.

${ }^{11}$ For some unknown reason, we have experienced lead problems with thicker gauge wires, so we recommend the use of gauge 40 or thinner gauge wires.

${ }^{12} \mathrm{D}$. Shoenberg, Magnetic Oscillations in Metals (Cambridge University Press, Cambridge, 1984).

${ }^{13}$ M. Lang, R. Modler, F. Steglich, N. Toyota, and T. Sasaki, Physica B 194-196, 2005 (1994). 